\title{
Ajuste de las propiedades mecánicas de la madera estructural por cambios del contenido de humedad
}

\author{
V. Rubén Ordóñez Candelaria ${ }^{1}$ \\ Raymundo Dávalos Sotelo ${ }^{1}$
}

\begin{abstract}
RESUMEN
El contenido de humedad presente en la madera y la variación del mismo es uno de los factores que más influencia tiene sobre las propiedades de la madera. Por lo tanto, es necesario conocer las proporciones en que varían las propiedades mecánicas cuando cambia el contenido de humedad. Utilizando resultados de estudios realizados por Madsen y escogiendo la especie más parecida en valores de resistencia y densidad a los pinos mexicanos se derivaron las expresiones para ajustar los resultados del estudio nacional sobre la resistencia de madera de coníferas. En este trabajo se presenta el procedimiento utilizado para obtener las expresiones de ajuste, que se emplearon en el estudio mencionado, para los datos de las pruebas de flexión, tensión, compresión paralela a la fibra y cortante paralelo a la fibra a un contenido de humedad uniforme, para derivar los valores de resistencia básicos para diseño.
\end{abstract}

PALABRAS CLAVE:

Contenido de humedad, resistencia mecánica, módulo de elasticidad, pinos mexicanos, Hem-Fir.

\begin{abstract}
The moisture content of wood, and its variation with atmospheric changes, is one of the factors with the greatest influence on wood properties. Therefore, it is necessary to know the degree to which mechanical properties vary as moisture content changes. Using the results from research conducted by Madsen, and selecting the species most similar to Mexican pines in strength and density, expressions were derived to adjust the results obtained in the national study of the strength of coniferous wood. In this paper, we present the procedure used to obtain the adjustment expressions for bending, tension, compression parallel to grain and shear parallel to grain. Moisture content was adjusted to a uniform value in order to obtain comparable strength values for design.
\end{abstract}

KEY WORDS:

Moisture content, strength, modulus of elasticity, Mexican pines, Hem-Fir.

1 Investigadores. Departamento de Productos Forestales y Conservación de Bosques. Instituto de Ecologia, A.C. Apdo. Postal 63. 91000 Xalapa, Ver., México.

Manuscrito recibido para su publicación el 5 de Abril de 1995 


\section{INTRODUCCION}

En los primeros trabajos realizados para investigar el efecto del contenido de humedad en la resistencia de la madera, se utilizaron pequeñas probetas libres de defectos, y a partir de sus resultados se establecieron las ecuaciones de ajuste que se presentan en las normas ASTM, (1993a) y en la referencia FPL (1987), tales como las que se presentan a continuación,

De ASTM, (1993a):

$$
P_{2}=P_{1} \frac{\left(\alpha-\beta M_{2}\right)}{\left(\alpha-\beta M_{1}\right)}
$$

Donde:

$\mathrm{P}_{2}=$ propiedad al contenido de

$\mathrm{P}_{1}=$ propiedad al contenido de humedad $\mathrm{M}_{1}$

$\mathrm{M}_{1}=$ contenido de humedad al que se determina la propiedad $\mathrm{P}_{1}$, en $\%$

$\mathrm{M}_{2}=$ contenido de humedad al que

se ajusta la propiedad \# $22 \%$

", \$ = coeficientes para las diferentes propiedades de resistencia, tabla 1

Tabla 1. Valores de las constantes para utilizar la ecuación 1

\begin{tabular}{||l|c|c||}
\hline \multicolumn{1}{|c|}{ PROPIEDAD } & " & $\$$ \\
\hline Módulo de elasticidad & 1.44 & 0.0200 \\
Módulo de ruptura & 1.75 & 0.0333 \\
Tensión paralela a la fibra & 1.75 & 0.0333 \\
Compresión paralela a la & 2.75 & 0.8330 \\
fibra & \\
Compresión perpendicular & 1.00 & 0.0000 \\
a la fibra \\
Cortante paralelo a la fibra & 1.33 & 0.0167 \\
\hline
\end{tabular}

Del Wood Handbook, FPL (1987):

$$
P=P_{12}\left(\frac{P_{12}}{P_{g}}\right)^{\frac{12-M}{M_{p^{-}}-12}}
$$

Donde:

$$
\begin{aligned}
P_{12}= & \text { propiedad al } 12 \% \text { de contenido } \\
& \text { de humedad } \\
M= & \text { contenido de humedad al que se } \\
& \text { desea ajustar } \\
M_{p}= & \text { contenido de humedad a partir } \\
& \text { del cual las propiedades no } \\
& \text { sufren cambios, cercano al Punto } \\
& \text { de Saturación de la Fibra (usar } \\
& 25 \% \text { si no se conoce el } \\
& \text { verdadero valor) } \\
P_{g}= & \text { propiedad de la madera en } \\
& \text { condición verde, CH } \$ \mathrm{M}_{\mathrm{p}}
\end{aligned}
$$

La ecuación 2 no es aplicable con carga máxima, impacto en flexión y tensión perpendicular a la fibra. Lo que se encontró para pequeñas probetas no representa la realidad de lo que sucede con la madera de tamaño estructural, cuyo comportamiento difiere del de pequeñas probetas, principalmente porque los defectos y otras características de la madera estructural (grietas, rajaduras, torceduras, etc.) generan cambios en el material, con variaciones del contenido de humedad.

En la norma ASTM D-1990, (ASTM, $1993 b)$ se establecen las siguientes ecuaciones de ajuste por cambios de contenido de humedad, para los valores de resistencia en flexión (MOR) y en tensión y compresión paralela a la fibra (UTS y UTC):

$$
\mathrm{S}_{2}=\mathrm{S}_{1}
$$

Cuando:

MOR \#170 kg/cm² (2415 psi)

UTS \#221 kg/cm² (3150 psi)

UTC \# 98 kg/cm² (1400 psi) 


$$
S_{2}=S_{1}+\left(\frac{S_{1}-B_{1}}{B_{2}-M_{1}}\right)\left(M_{1}-\right)
$$

Cuando:

MOR $>170 \mathrm{~kg} / \mathrm{cm}^{2}(2415 \mathrm{psi})$

UTS > $221 \mathrm{~kg} / \mathrm{cm}^{2}$ (3150 psi)

UTC > $98 \mathrm{~kg} / \mathrm{cm}^{2} \quad(1400 \mathrm{psi})$

Donde:

$\mathrm{S}_{1}=$ valor de la propiedad al contenido de humedad 1

$\mathrm{S}_{2}=$ valor de la propiedad al contenido de humedad 2

$\mathrm{M}_{1}=$ contenido de humedad 1

$\mathrm{M}_{2}=$ contenido de humedad 2

$\mathrm{B}_{1}, \mathrm{~B}_{2}=$ constantes de la tabla 2

Tabla 2. Valores de los coeficientes B1 y B2 para aplicar la ecuación 3

\begin{tabular}{||c|r|r|r||}
\hline \hline COEFICIENTE & MOR & \multicolumn{1}{c|}{ UTS } & \multicolumn{1}{c|}{ UTC } \\
\hline B1 & 170.0 & 221.0 & 98.0 \\
B2 & 2.8 & 5.6 & 2.4 \\
\hline
\end{tabular}

Este procedimiento de las normas ASTM D-1990 se derivó con base en estudios de la madera de Douglas Fir y Southern Pine. Es de notar que los valores límite a partir de los cuales cambia la resistencia son similares a los valores de las rectas con pendiente cero o positiva, como se observa en la figura 1, la línea del 5 percentil.

En este trabajo se presentan las ecuaciones utilizadas para ajustar los resultados de las pruebas realizadas en el proyecto de "Calificación y clasificación de la madera de pino para usos estructurales, Fase III", Echenique et al., (1987). Es importante destacar que los resultados de esta investigación requieren de más trabajo experimental para poder llegar a un procedimiento mucho más refinado con el cual se pueda ajustar la resistencia de la madera por cambios en su contenido de humedad.

El muestreo para este proyecto se realizó tomando piezas de la producción diaria de los aserraderos incluidos en el estudio. En algunos casos, la madera fue secada en estufa y en otros el secado se realizó al aire libre. Este último procedimiento genera entre las piezas una alta variabilidad entre los valores de contenido de humedad. Para la definición de los esfuerzos básicos de resistencia de la madera debe, de ser posible, determinarse su resistencia a un valor fijo de contenido de humedad. De no lograrlo, será necesario ajustar los resultados de las pruebas de resistencia al valor de $\mathrm{CH}$ escogido; en este caso, el ajuste fue al $18 \%$ de contenido de humedad. Pero, para hacer comparaciones en la derivación de los valores de la investigación se manejaron los datos a un contenido de humedad del $12 \%$, los que posteriormente se modificaron al contenido de humedad del $18 \%$, que es el definido por el reglamento de Construcciones del D.F. para distinguir madera seca y húmeda (RCDF, 1987).

No existen estudios con piezas estructurales de especies coníferas mexicanas, que permitan conocer el comportamiento de la resistencia de la madera al variar su contenido de humedad. Es por esto, que los ajustes de los resultados se definieron con información generada en el extranjero, presumiendo que los pinos mexicanos se comportan de manera similar al grupo que se escogió para derivar las ecuaciones de ajuste, que es el Hem-Fir, grupo con una densidad (peso anhidro/volumen verde) de 0.41 (ASTM, 1992), similar a la de los pinos mexicanos, 0.43 (Bárcenas y Dávalos, 1996). 


\section{PROCEDIMIENTO}

Los datos utilizados se tomaron del trabajo de Madsen, et al., (1980), correspondientes al grupo Hem-Fir del tamaño nominal de $2 \times 6$ plg y clase No. 2 y mejor; esta clase estructural es similar a la calidad de la madera que se muestreó para el proyecto. A partir de estos datos, se obtuvieron las gráficas de variación de la resistencia de la madera con respecto a su contenido de humedad, como se ilustra en la gráfica de la figura 1.

Ajuste de la resistencia en flexión

Con los datos de Madsen et al., (1980), se derivaron las expresiones para ajustar los resultados de las pruebas de flexión a un valor correspondiente al $12 \%$ de $\mathrm{CH}$ utilizando los valores del grupo Hem-Fir tal como se muestra en la tabla 3 para el Módulo de Ruptura.

Con los datos de las dos últimas columnas de la tabla 3 , se obtuvo la regresión que mejor se ajusta a los datos, resultando en una expresión logarítmica como la siguiente:

$$
x=-\frac{1}{0.53} \operatorname{Ln} \frac{y}{283.3}
$$

Con un valor de $r^{2}=0.994$. Esta expresión se puede reescribir con algunos cambios:

$$
\begin{array}{ll}
\mathrm{Si}: & \\
\mathrm{k} & =\text { porcentaje de variación } \\
\mathrm{y} & =\operatorname{MOR}_{12} \text { (Módulo de Ruptura en } \\
& \text { Flexión) }
\end{array}
$$

La ecuación de la gráfica se puede reescribir como:

$$
k=-\frac{1}{0.53} \operatorname{Ln} \frac{M_{12}}{283.3}
$$

El valor de k se obtuvo de la siguiente manera:

$$
k=\frac{\left(M O R-M O R_{12}\right)}{M O R_{12}} \frac{10 C}{\left(C H^{-}\right.}
$$

de donde:

$$
k \frac{C H-12}{100}=\frac{M U K}{M O R_{12}}-1
$$

Sustituyendo la ecuación (5) en la (7) y despejando tenemos:

$$
M O R_{C H}=M O R_{12}\left(1-\left(\frac{C H-12}{53}\right) \operatorname{Ln} \frac{M O R_{12}}{283.3}\right)
$$

donde:

$\begin{aligned} \text { MOR }_{12}= & \text { módulo de ruptura en flexión } \\ & \text { a } 12 \% \text { de contenido de } \\ & \text { humedad }\end{aligned}$

$\mathrm{MOR}_{\mathrm{CH}}=$ módulo de ruptura en flexión a un contenido de humedad, $\mathrm{CH}$

$\mathrm{CH}=$ contenido de humedad de la pieza al momento de la prueba

Con la ecuación (8) se puede ajustar la resistencia en flexión de la madera desde cualquier contenido de humedad al $12 \%$.

En las figuras 2 y 3 se observan los datos experimentales y la curva ajustada de los valores de $\mathrm{k}$ a diferentes valores del Módulo de Ruptura y del Módulo de Elasticidad. Con el mismo procedimiento y utilizando los datos de Madsen (1982) se obtuvieron las expresiones para el Módulo de Elasticidad en flexión, resistencia en compresión y tensión paralela a la fibra y cortante paralelo a la fibra, que se presentan a continuación: 


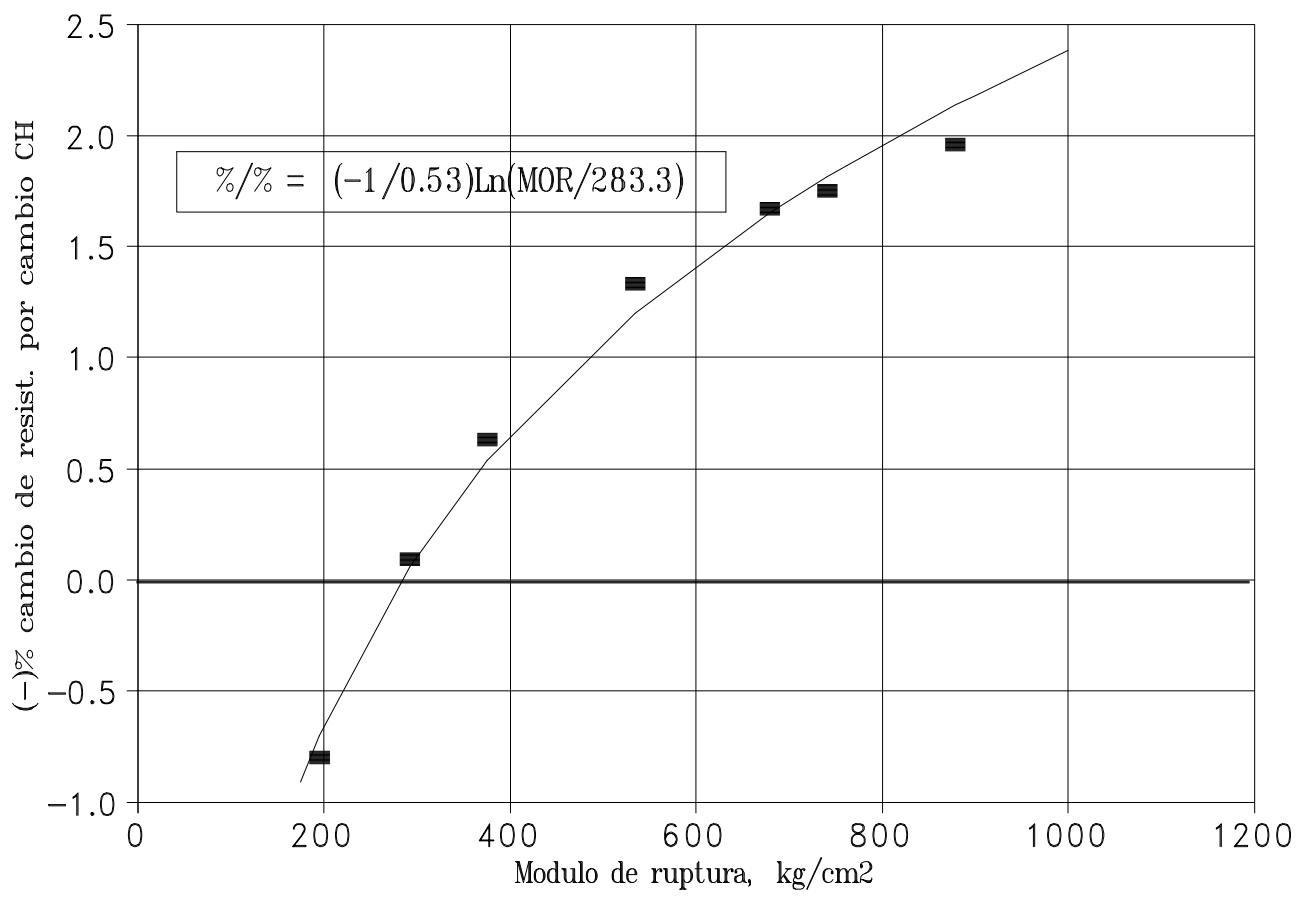

Figura 1. Variación de la resistencia en flexión por cambios de contenido de humedad, de madera de hem-Fir, a diferentes niveles de resistencia (Madsen et al., 1980).

Tabla 3 . Variación de la resistencia en flexión de la madera de Hem-Fir, por variación del $\mathrm{CH}$. Normalizada al 12.

\begin{tabular}{||c|c|l|l|c||}
\hline \hline PERCENTIL & ECUACION & MOR $_{\mathrm{CH}=21.4}$ & MOR $_{\mathrm{CH}=12}$ & $\mathrm{k},(\% / \%)$ \\
\hline 5 & $\mathrm{y}=1.560 \mathrm{x}+176.35$ & 209.70 & 195.10 & 0.80 \\
15 & $\mathrm{y}=-2.550 \mathrm{x}+295.13$ & 289.60 & 292.10 & -0.09 \\
25 & $\mathrm{y}=-2.378 \mathrm{x}+404.98$ & 354.10 & 376.50 & -0.63 \\
50 & $\mathrm{y}=-7.107 \mathrm{x}+619.75$ & 467.61 & 534.50 & -1.33 \\
75 & $\mathrm{y}=-11.386 \mathrm{x}+816.65$ & 573.00 & 680.00 & -1.67 \\
85 & $\mathrm{y}=-12.948 \mathrm{x}+896.38$ & 619.20 & 741.00 & -1.75 \\
95 & $\mathrm{y}=-17.248 \mathrm{x}+1085.88$ & 716.70 & 878.90 & -1.96 \\
\hline
\end{tabular}




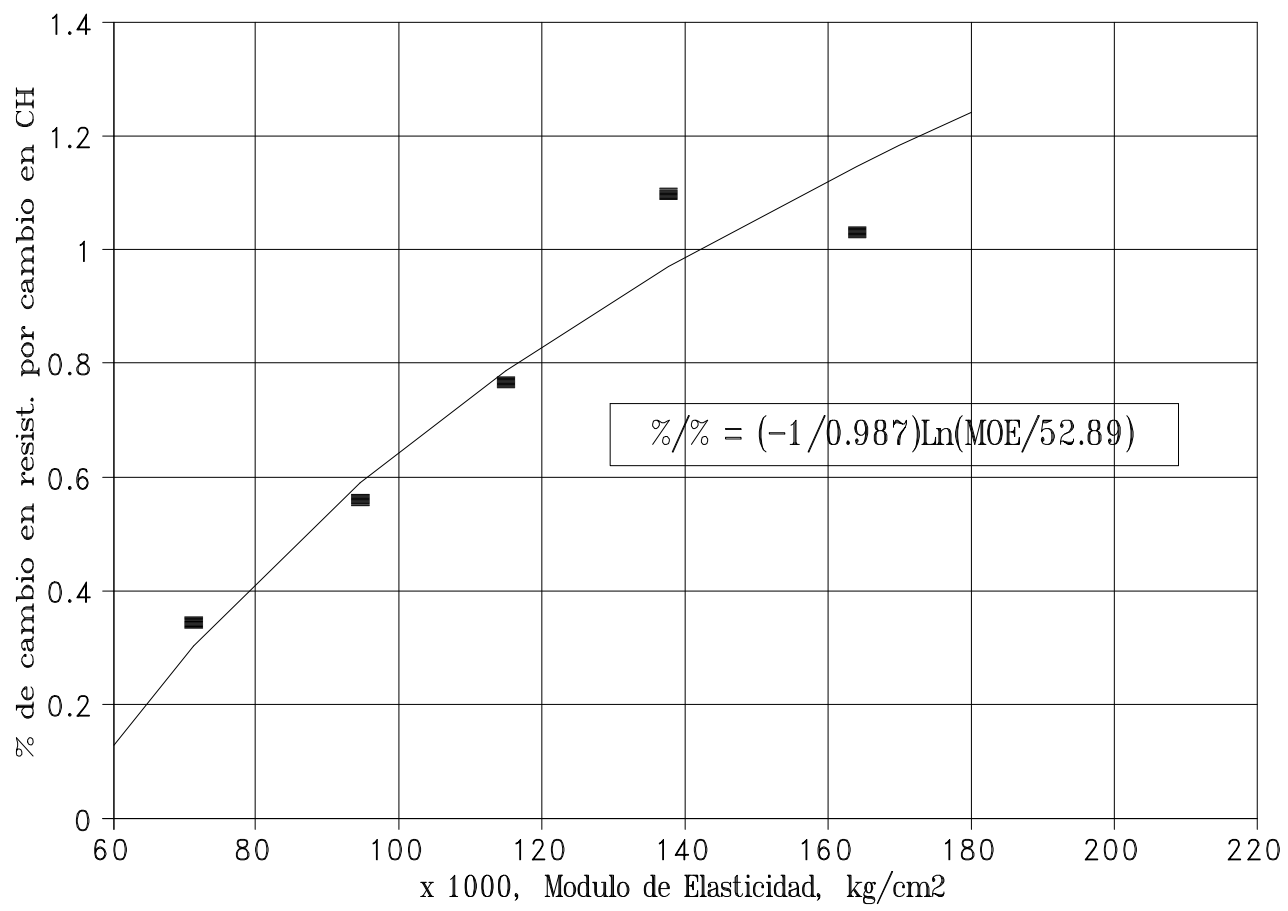

Figura 2. Porcentaje de cambio de la resistencia en flexión por cambio en el contenido de humedad, según el nivel de resistencia. Normalizada a $\mathrm{CH}=12 \%$

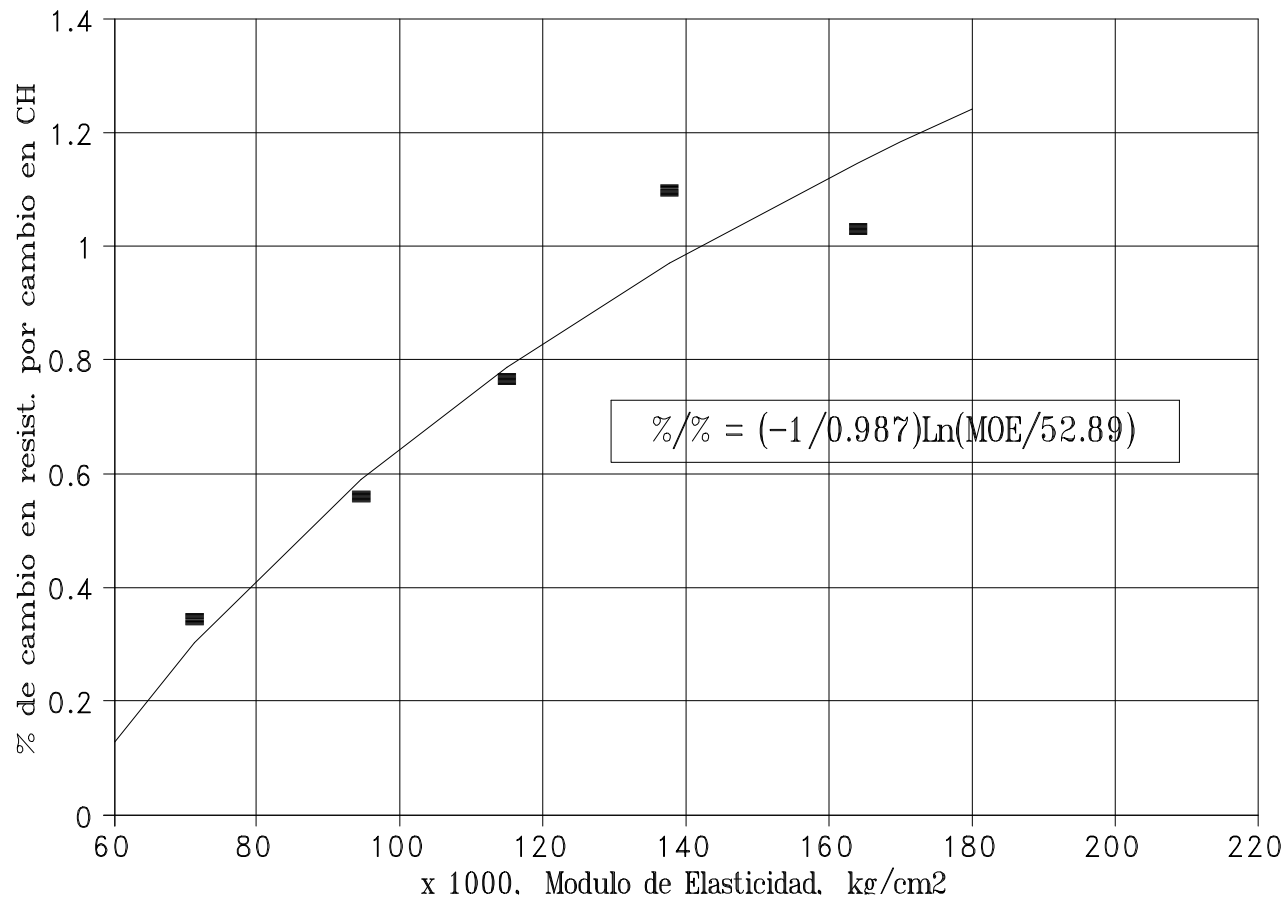

Figura 3. Porcentaje de cambio en el Módulo de Elasticidad en flexión por cambio del contenido de humedad. Normalizada a $\mathrm{CH}=12 \%$ 


\section{Ajuste del Módulo de Elasticidad (MOE)(9):}

$$
M O E_{C H}=M O E_{12}\left(1-\left(\frac{C H-12}{98.7}\right) \operatorname{Ln} \frac{M O E_{12}}{52.89}\right)
$$

\section{Ajuste de la resistencia en compresión paralela a la fibra:}

$$
F c_{C H}=F c_{12}\left(1-\frac{C H-12}{75} \operatorname{Ln} \frac{F c_{12}}{64.5}\right)
$$

\begin{abstract}
Ajuste de la resistencia en tensión paralela a la fibra:

El ajuste en este caso presenta una variante debido a que los datos experimentales de Madsen (1982) indican una tendencia diferente antes y después de un $\mathrm{CH}=20 \%$. El ajuste consiste en determinar el valor de la resistencia a un contenido de humedad del $20 \%$, si el dato experimental se determinó a un $\mathrm{CH}>$ $20 \%$, utilizando la ecuación (12), y con este valor se calcula el valor de la resistencia a un $\mathrm{CH}=12 \%$. Si el $\mathrm{CH}$ de la probeta ensayada fue menor 0 igual al $20 \%$ se utiliza directamente la ecuación (11) para calcular el valor de la resistencia a un $\mathrm{CH}=12 \%$.
\end{abstract}

$t_{C H \leq 20}=F t_{12}\left(1-\left(\frac{C H-12}{101}\right) L n \frac{F t_{12}}{902}(11)\right.$

Si CH \#20\%

$$
F t_{C H>20}=F t_{20}\left(1-\frac{C H-20}{9.5} \operatorname{Ln} \frac{F t_{20}}{150}\right)
$$

Si $\mathrm{CH}>20 \%$

\section{Ajuste de la resistencia en cortante} paralelo a la fibra:

Utilizando datos de Madsen y Nielsen (1978):

$$
F v_{C H}=F v_{12}\left(1-\frac{C H-12}{0.40} \operatorname{Ln} \frac{F v_{12}}{3.44}\right)
$$

\section{CONCLUSIONES}

Con las expresiones aquí citadas se pueden realizar los ajustes de los resultados de las pruebas con una aproximación aceptable, sin que se incurra en errores muy grandes, a pesar de haber usado los datos de especies de coníferas diferentes a las nacionales. Debe tenerse en cuenta que son útiles para especies similares a las utilizadas en su desarrollo y aplicación; para especies muy diferentes deberá hacerse un análisis similar al de este trabajo para poder derivar las expresiones de ajuste.

Es importante señalar que este modelo no es un modelo reversible, lo cual es una desventaja, aunque en este caso, las diferencias que se presentan al emplearlo, son menores a $1 \%$, por tratarse de valores bajos de resistencia. Para valores mayores, las diferencias sí son sensibles (pueden ser mayores a $5 \%$ para valores mayores a $500 \mathrm{~kg} / \mathrm{cm}^{2}$ )

Todos los análisis de resultados del proyecto "Calificación y clasificación de la madera de pino para usos estructurales, Fase III" (Echenique et al., 1987) se hicieron con los valores de la resistencia y módulo de elasticidad en flexión ajustados al 12\%. Estos análisis incluyeron: efecto del tamaño, diferencias regionales y diferencias por época de muestreo. Para la determinación de los valores de diseño se siguió el procedimiento indicado a continuación:

Los valores de las propiedades mecánicas en flexión y tensión paralela para las coníferas mexicanas ajustadas al $12 \%$, fueron los mismos que se emplearon para derivar los esfuerzos de diseño al $18 \%$, porque de acuerdo con la gráfica 1 y ecuaciones de la tabla 3 , valores menores a $400 \mathrm{~kg} / \mathrm{cm}^{2}$ no sufren una variación en resistencia mayor al 
Ajuste por contenido de humedad. Ordóñez y Dávalos

$3.8 \%$ e inclusive al nivel del 50 percentil sería necesario aumentar los valores de esfuerzos en lugar de disminuirlos (la pendiente de la recta a ese nivel es positiva, es decir, a mayor contenido de humedad, mayor resistencia).

En el caso de los resultados en compresión paralela a la fibra, los valores ajustados al $12 \%$, sí fueron corregidos, puesto que las diferencias en resistencia para esta propiedad si son significativas, cuando varía el $\mathrm{CH}$ aún a niveles bajos de resistencia. El procedimiento adoptado en este caso fue el siguiente: los valores determinados para un $\mathrm{CH}=12 \%$ fueron disminuidos $2.17 \%$ por cada porciento de cambio en $\mathrm{CH}$. Es decir, se redujeron $13 \%$ $(2.17 \times 6)$, considerando una variación lineal de la resistencia.

La pendiente de la recta de ajuste para la madera de las dos clases de resistencia reconocidas por el Reglamento (RCDF, 1987) es la misma, de ahí que el ajuste sea proporcional en ambos casos. Los valores especificados en el Reglamento actual reflejan estas modificaciones.

El modelo de ajuste empleado es uno de los existentes en la literatura y fue el que se consideró el más apropiado para la madera de pinos mexicanos, para guardar una consistencia en la filosofía de la pruebas, similar a la propuesta por Madsen.

Existen otros modelos, aparte de los ya mencionados de la ASTM; entre los más importantes en Norte América se pueden citar los de Green (1980) y Green y Evans (1992).

\section{RECONOCIMIENTOS}

Este trabajo fue realizado con fondos fiscales asignados al Departamento de Productos Forestales y Conservación de
Bosques del Instituto de Ecología, A.C. con la clave 902-13.

\section{REFERENCIAS}

ASTM. 1992. Standard test methods for establishing clear wood strength values. ASTM D2555-93. Vol. 04-09 Ph. EUA.

ASTM. 1993a. Standard practice for evaluating allowable properties for grades of structural lumber. ASTM D2915-90. Vol. 04-09 Ph. EUA.

ASTM. 1993b. Standard practice for establishing allowable properties for visually-graded dimension lumber from in-Grade tests of full-size specimens. ASTM D1990-91. Vol. 04-09 Ph. EUA.

Bárcenas P., G. y R. Dávalos S. 1996. Shrinking and sw elling characteristics of 103 Mexican woods. Submitted to be published to J ournal of Tropical Forest Products. $36 \mathrm{p}$.

DDF. 1987. Normas técnicas complementarias para diseño y construcción de estructuras de madera. Gaceta Oficial del Departamento del Distrito Federal, Quinta época, Núm. 48. México, D. F. $43 \mathrm{p}$.

Echenique M., R., R. Dávalos S. y F. Robles, F-V. 1987. Calificación y clasificación de la madera de pino para usos estructurales. Fase III. Informe Final al CIID. INIREBLACITEMA. Xalapa, México.

FPL. 1987. Wood handbook: Wood as an engineering material. Agriculture Handbook 72 Washington, D.C. US Department of Agriculture. 466 p. 
Green, D.W. 1980. Adjusting the static strength of lumber for changes in moisture content. Workshop of research needs on effect of the environment on design properties of lumber. Forest Products Laboratory. USDA. Madison Wi. EU

Green, D.W. y J.W. Evans. 1992. Moisture content adjustment procedures for engineering standards. Proceedings, 25th Meeting of International Council for Building Structures. Suecia.

Madsen, B. y P.C. Nielsen. 1978. InGrade testing investigation of test parameters in longitudinal shear:
Structural Research Series Report No. 23. Department of Civil Engineering, University of British Columbia, Vancouver, Canadá.

Madsen, B., W. J anzen, y J. Zwaagstra. 1980. Moisture effects in lumber. Struct. Research Series Paper No. 27, University of British Columbia, Department of Civil Engineering. Vancouver, Canadá.

Madsen, B. 1982. Recommended moisture adjustment factors for lumber stresses. Canadian J ournal of Civil Engineering 9(4):602-610. 